\title{
Cytokine removal in human septic shock: Where are we and where are we going?
}

\author{
Patrick M. Honore ${ }^{1 *}$, Eric Hoste ${ }^{2}$, Zsolt Molnár ${ }^{3}$, Rita Jacobs ${ }^{4}$, Olivier Joannes-Boyau ${ }^{5}$, Manu L. N. G. Malbrain ${ }^{4,6}$ \\ and Lui G. Forni ${ }^{7,8}$
}

\begin{abstract}
Although improving, the mortality from septic shock still remains high despite increased international awareness. As a consequence, much effort has focused on alternative treatment strategies in an effort to improve outcomes. The application of blood purification therapies to improve immune homeostasis has been suggested as one such method, but these approaches, such as high-volume continuous haemofiltration or cytokine and/or endotoxin removal, have enjoyed little success to date. More recently, the use of sorbent technologies has attracted much attention. These adsorbers are highly effective at removing inflammatory mediators, in particular, cytokines, from the bloodstream. This narrative review is the executive summary of meetings held throughout the 6 th International Fluid Academy Days in Antwerp, Belgium (Nov 23-25, 2017), focusing on the current understanding regarding the use of such adsorbers in humans with septic shock. We followed a modified Delphi approach involving a combination of evidence appraisal together with expert opinion in order to achieve recommendations for practice and, importantly, future research.
\end{abstract}

Keywords: Blood purification, Cytokines, Sepsis, Septic shock, Haemoperfusion, Cytosorb, Sorbents, Cartridges, Immune modulation, DAMPS, PAMPS

\section{Introduction}

The pathogenesis of sepsis: blood as "biopsy" of tissue inflammation

Septic shock continues to have significant mortality [1]. The underlying pathophysiology is complex with both pathogenic and host factors (PAMPs (pathogen-associated molecular patterns) and DAMPs (damage-associated molecular patterns)) playing a significant role in the development and subsequent outcome [2]. However, the heterogeneity of septic shock prevents adequate characterization of patients and may hinder subsequent clinical intervention(s). Sepsis and septic shock affect anywhere between 100 to 1000 per 100,000 person-years and 19 per 100,000 person-years depending on the cohort studied [3-6], with reported mortality rates ranging between 20 to $50 \%$ [7-11]. Moreover, the reported incidence is

\footnotetext{
*Correspondence: Patrick.Honore@CHU-Brugmann.be

1 Intensive Care Department, CHU Brugmann University Hospital, 4, Place Arthur Van Gehuchtenplein, 1020 Brussels, Belgium

Full list of author information is available at the end of the article
}

increasing, although this may be attributed to reporting bias in so-called claims-based databases, as data analysis on electronic health records cannot confirm this trend [12-15]. Therefore, it is of no surprise that the treatment of sepsis has become a major global health issue. Indeed, in the USA during 2011 sepsis accounted for just over 5\% of total hospital costs corresponding to $\$ 20$ billion dollars [16].

\section{The place for blood purification}

Patients with sepsis are often treated in areas of intensive care given that close monitoring and intense therapeutic support are needed [17]. Early treatment includes the use of timely, appropriate antibiotics, intravenous fluids, oxygen therapy as well as vasopressor and inotropic support where needed. Other additional treatments including extracorporeal or so-called blood purification techniques (BPT) have also been tried [18]. These techniques include (among others): haemofiltration, haemoperfusion, intermittent or continuous high-volume haemofiltration (HVHF), plasmapheresis or adsorption. The rationale 
behind such an approach is to achieve "immune homeostasis" which theoretically reduces the potential damage caused by dysregulation of the host response to infection. This may be heralded by a profound rise in inflammatory mediators including cytokines which contribute to the dramatic systemic effects of sepsis, mainly in septic shock $[9,19]$. The recently updated Sepsis 3.0 consensus definitions state that sepsis is an infection accompanied by life-threatening organ dysfunction caused by a dysregulated host response [20]. Given the pivotal role of cytokine production in sepsis, it follows that removal of these substances, through such BPT, may attenuate the response particularly in the early phase of sepsis [21]. Several hypotheses have been proposed as to the potential mechanisms underpinning potential benefit. These include cytotoxic theories including the peak concentration hypothesis whereby all inflammatory mediators are removed at a given rate, dependent on the BPT used and assuming they are filtered [21-24]. Alternatively, the cytokinetic theory proposes that cytokines are removed, thereby creating a cytokine gradient between the bloodstream and tissues allowing leucocyte-enhanced trafficking [25]. In the same line, cytokine levels can also be seen as communicating messengers to talk to cells, recruit some, depress others and reduce cell metabolism for others [22]. Despite early promise, no multicentre randomized controlled studies have demonstrated a survival benefit including the use of HVHF where higher flows may lead to increased cytokine removal were tried (the cytotoxic threshold immune modulation hypothesis) [26-28]. Other extracorporeal blood purification therapies also have failed with significant outcome data lacking with no treatment demonstrating a translatable survival benefit in any randomized controlled study [29-31]. This somewhat too simplistic view takes into account several new concepts. Indeed, blood level of mediators (more than cytokines) implies the saturation of the interstitial and cellular compartments to be present in the blood. It is the so-called tip of the iceberg theory [31]. This theory is very similar to the "threshold immune modulation theory" [23]. In addition, plasma mediator levels depend on several factors: the intensity of production, the number of cell receptors availability, the clearance of such mediators, the affinity of the receptors for such mediators. As an example, the interleukin (IL)- 6 receptor is an agonist one, which differs from tumour necrosis factor (TNF)-soluble receptors and IL-1 receptors that are inhibitory; the consequence is that a low IL-6 level with a high level of receptor induces more cellular response than high level of IL-6 with a low level of receptors [22]. This very important issue is showing that an IL-6 level alone may not be very predictive of the future response of the organism. DAMPs are also playing a key role and especially the endosomal DAMPs that are eliminated via the BPT and are capable of inducing cellular damage and apoptosis [32]. This may explain the initial "positive" observational trials with HVHF. The reported improvement in hemodynamic status associated with or not with lactate reduction might mainly be the result of the fluid replacement therapy made with large volumes of crystalloids containing high buffer concentrations. As a consequence, the induced $\mathrm{pH}$ increase might have changed the affinity for catecholamines to their receptors improving hypotension [28-30].

\section{Impact on mortality}

Extracorporeal haemoperfusion with Polymyxin B (PMXHP) has shown improvement in organ dysfunction and a survival benefit in small studies [33] including a small randomized trial [34], while larger trials failed to confirm these findings $[35,36]$. Evaluating the use of PMX-HP in a randomized controlled trial of adults treated for endotoxemia and septic shock (the EUPHRATES study) included patients with persistent septic shock despite adequate fluid resuscitation and vasopressor treatment [37]. An endotoxin activity assay (EAA) was applied. No mortality difference was observed in the "per protocol population" $(n=244$, multiple organ dysfunction score (MODS) $>9$, $E A A \geq 0.6)$. However, among the patients in refractory shock with MODS of more than 9 and an EAA between 0.6 and 0.9 , a significant $10.7 \%$ reduction in 28 -day mortality in a post hoc analysis was recognized after receiving two sessions of PMX-B-HP [38]. Post-hoc analysis revealed a significant mortality reduction when the EAA was limited to less than 0.9 , which may suggest an upper limit to a pre-treatment endotoxin burden under these treatment conditions [38]. A recent meta-analysis of 17 trials demonstrated that PMX-B-HP treatment may reduce mortality in patients with severe sepsis and septic shock. The included studies were stratified into three groups based on the mortality rates of the conventional treatment group: low-risk group (mortality rate $<0.3$ ), intermediate-risk group (0.3-0.6) and high-risk group (>0.6). Risk ratios with 95\% confidence intervals (CI) for the mortality-stratified analysis between the PMX-HP and conventional treatment groups were calculated and presented as summary statistics. This a posteriori classification can be seen as a major caveat of this meta-analysis. Also mortality rate does not reflect cytokine concentrations and load and therefore is not a predictor of response to cytokine removal. Disease severity subgroup metaanalysis revealed a significant risk reduction in overall mortality in the intermediate-risk (mortality rate $0.3-0.6$ ) and high-risk (mortality rate $>0.6$ ) groups, but not in the low-risk (mortality rate $<0.3$ ) group reinforcing the view 
that rigorous patient selection is crucial for treatment success [39].

Lastly, two editorials set the pace by stating that while blood purification in sepsis remains a valid approach, the use of adsorbers and their current efficacy of endotoxin/ cytokine elimination cannot be recommended to reduce the mortality in absence of positive randomized controlled trials (RCTs) [40, 41].

\section{Cytokine storm and capillary leak}

Capillary leak represents the maladaptive and undesirable movement of fluid and electrolytes with or without protein into the interstitium that generates anasarca and end-organ oedema potentiating organ dysfunction and or failure [42]. The global increased permeability syndrome (GIPS) defined as a positive cumulative fluid balance and new onset organ dysfunction/failure is described in patients with persistent systemic inflammation resulting in continuing transcapillary albumin leakage. GIPS may represent the third phase in a continuum after the initial cytokine storm and ischaemia-reperfusion injury and could be a potential indication to start BPT [43].

\section{Finding the answers}

The focus of this consensus meeting was to determine whether a reduction in cytokine levels is possible through the use of sorbent technology by use of the CytoSorb ${ }^{\circledR}$ (Cytosorbents, Corporation, New Jersey, USA) device. This consists of a single-use haemoadsorption cartridge which can be used with standard blood pumps, such as those found on RRT machines or through a haemoperfusion device [44-46]. The CytoSorb cartridge is the only currently available CE-marked device shown to consistently lower excessive cytokines in severe sepsis. The cartridge is filled with sorbent beads made from a porous polymer that adsorbs and capture cytokines as blood passes through the device. This process is concentration dependent, and so the higher the levels of cytokines in the blood, the faster the levels are reduced. Although CytoSorb therapy has been described for other indications, including intoxications, rhabdomyolysis, hyperbilirubinemia, etc., we chose to concentrate on human studies in sepsis and septic shock.

\section{Consensus process}

This consensus meeting took place during the 6th International Fluid Academy Day (IFAD) held in Antwerp, Belgium, at the end of November (November 23-26, 2017). We followed a modified Delphi approach involving a combination of evidence appraisal together with expert opinion in order to achieve recommendations for practice and future research. A panel of clinicians representing intensive care, anaesthesia and critical care nephrology from Europe convened to discuss the issues related to cytokine haemoadsorption using the CytoSorb $^{\circledR}$ or other devices in humans with sepsis and septic shock. The participants were tasked with summarizing the literature in humans to date, highlighting knowledge gaps and recommending areas of potential research. Reviews of the literature were performed prior to the meeting with a presentation to the participants and consensus was reached. MEDLINE ${ }^{\circledR}$ and PubMed searches were performed using the search terms "cytosorb", "haemofiltration", "haemoadsorption", "haemoperfusion" and "sepsis OR septic shock OR critical care OR critical illness"'. The reference lists of identified papers were screened to identify other relevant papers. Where necessary, further discussion was performed after the meeting. Although representatives from industry were present, they did not contribute to the discussion unless specifically questioned and did not play a role in the manuscript preparation. This paper serves as the final Executive Summary of the meeting.

\section{Pathophysiology}

As discussed, the inflammatory state associated with sepsis leads to release into the circulation of many proinflammatory mediators leading to deleterious systemic effects [44]. Although this effect may in part be modified through anti-inflammatory mediators, sustained effects may lead to relative immunoparesis [47, 48]. This concept is important given the overused simplistic view that sepsis is associated with a significant uncontrolled release of pro-inflammatory mediators and simple modulation of these would translate into improved patient outcomes [21]. Hence the desire for a more specific targeted therapy, rather than just adoption of "standard" continuous renal replacement techniques as a profound pro-inflammatory response, may lead to dysregulation of the anti-inflammatory pathways, and the aim of immune homeostasis may be thwarted by a worsening of the host response (Figs. 1, 2). Indeed, such dysregulation can be seen in patients with sepsis who transition to a late anti-inflammatory phenotype referred to as sepsis-associated immunosuppression (SAI) [49-51]. This process involves the reduction in the production of the inflammatory mediators together with direct effects on antigen-presenting activity. In turn, this may lead to enhanced immune tolerance with changes demonstrated in circulating immune cells and within tissues [52]. Following injury or infection, there is activation of humoral factors such as complement that then trigger the antigen-presenting cells (APCs) that release a host of mediators including cytokines. In turn, these attract and, as a consequence, activate more APCs and neutrophils. Migration to draining lymph nodes stimulates further 


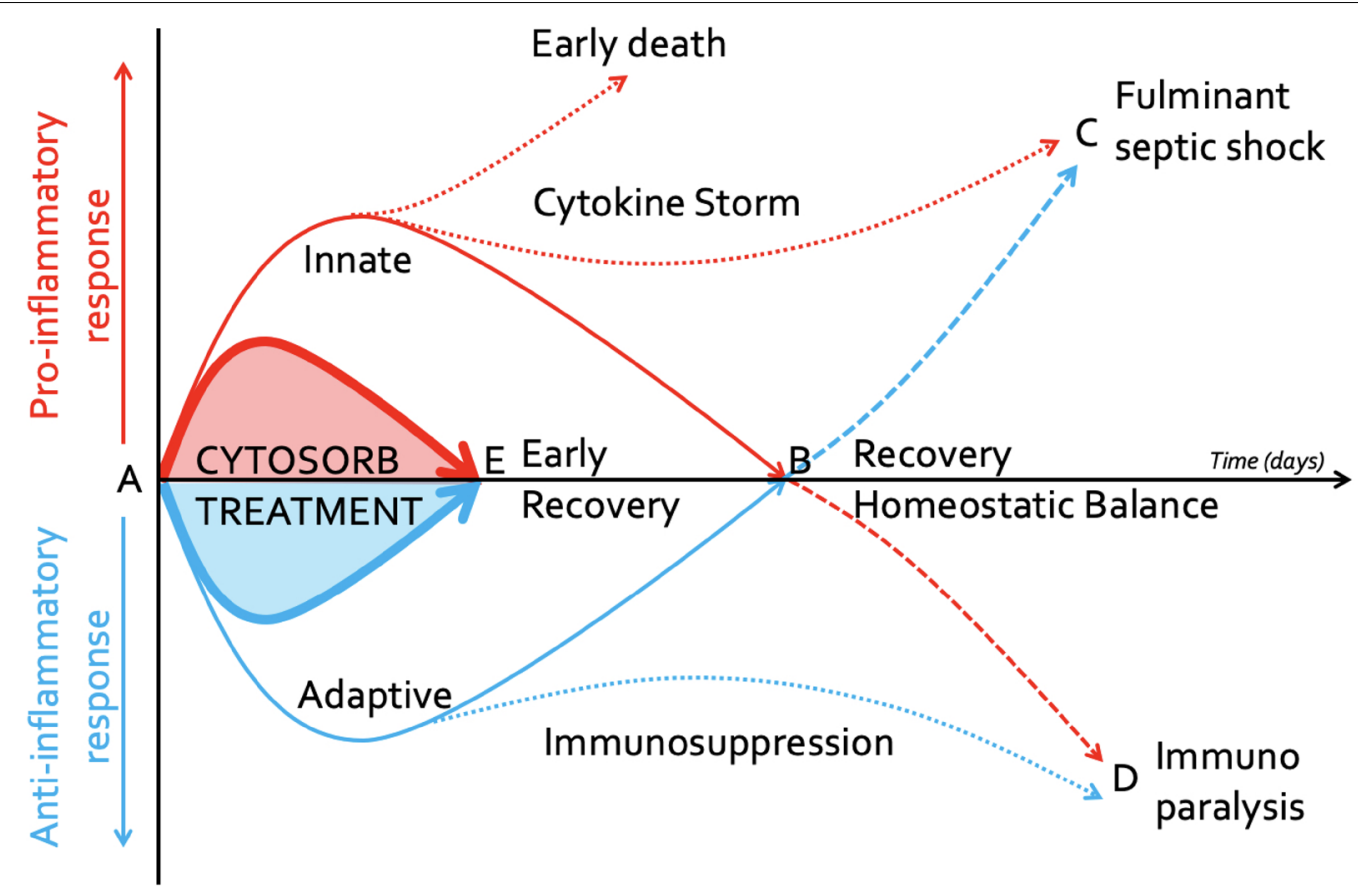

Fig. 1 Cytokine response after sepsis. Normal and abnormal immune response after an (infectious) insult (A). Recovery with regaining of the homeostatic balance occurs when pro-inflammatory (solid red line) and anti-inflammatory (solid blue line) mediators (B) return back to baseline levels. Early death or fulminant septic shock (C) can occur following early increased innate pro-inflammatory response (cytokine storm, dotted red line) or after initial adaptive immunosuppression (dashed blue line). Immunoparalysis (D) can occur following early increased adaptive anti-inflammatory response (immunosuppression, dotted blue line) or after initial pro-inflammatory response (dashed red line). Haemoadsorption with Cytosorb ${ }^{\circledR}$ may attenuate the initial pro- (bold red line) and anti-inflammatory (bold blue line) response resulting in early recovery ( $\mathrm{E}$ )

adaptive immune activity. These processes are followed by the anti-inflammatory response whereby there is a diminution of the host response as a consequence of anti-inflammatory cytokines such as IL-10 being produced. In the critically ill, this may lead to immunosuppression as described $[47,48]$. Cytokines play a pivotal role in the progression of the sepsis response. In early sepsis TNF- $\alpha$, a pro-inflammatory cytokine released by monocytes and macrophages, is a marker of early sepsis and enhances the adaptive immune response [53]. Several studies have demonstrated an association between mortality and elevated TNF- $\alpha$ although the utility of TNF- $\alpha$ as a predictor of mortality has been questioned [54]. IL-6, predominantly produced by monocytes and macrophages, induces $\mathrm{T}$-cell activation and $\mathrm{B}$ cell proliferation and stimulates the acute phase response all leading to augmentation of the immune response [55]. Circulatory levels rise rapidly after infection with peak levels approaching $2 \mathrm{~h}$ after insult with increased levels associated with poorer outcomes. However, cytokines may be derived from non-immune cells and have variable clearance rates and as such may not reflect immune cell functionality in all cases [56]. Furthermore, IL-6 has a relatively fast induction coupled with a short half-life, and these characteristics make it ideally suited for patient monitoring although to date most laboratories do not offer routine assay. With regard to anti-inflammatory cytokines, IL-10 is the most studied and has similar kinetics to IL-6. However, unlike IL-6, Il-10 induces antigen tolerance enhancing SAI and may predict mortality [57]. Obviously, considering IL-6 as a potential target remains interesting, but the profile of IL-6 kinetics in critically ill patients could be heterogeneous and explained by several factors. Indeed, in the abdomix study, some patients were with and without high levels of IL-6 also in the control arm $[35,36]$.

\section{Rationale for cytokine removal Rationale}

The enhanced inflammatory response seen in septic shock is associated with a high mortality $[57,58]$ correlated with the production of pro- and anti-inflammatory mediators [57] rather than disequilibrium between proand anti-inflammatory mediators [59] (Fig. 3). This has stimulated much effort towards potential attenuation of this response particularly as early studies suggested that continuous veno-venous haemofiltration $(\mathrm{CVVH})$ may reduce cytokine levels [60]. However, as discussed, these 


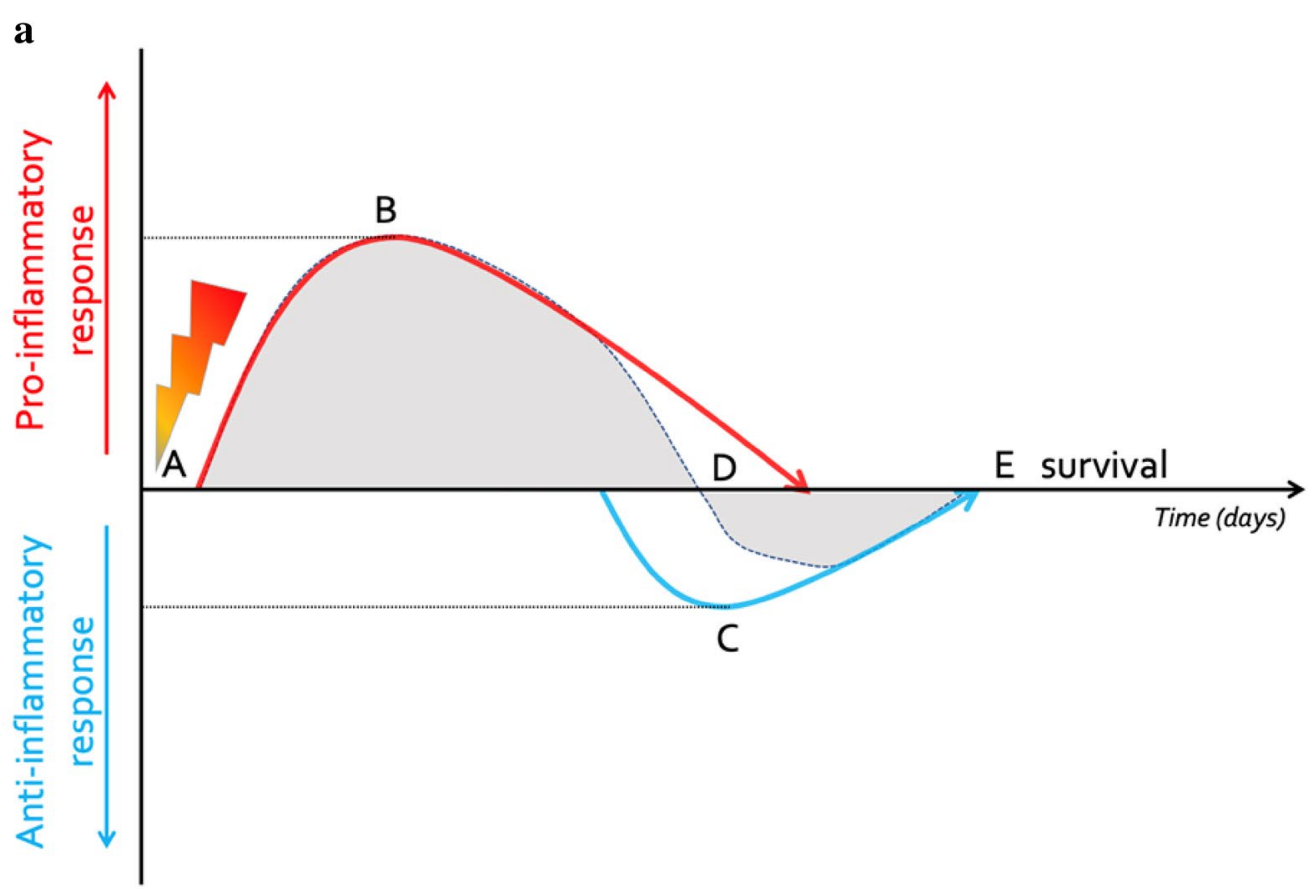

b

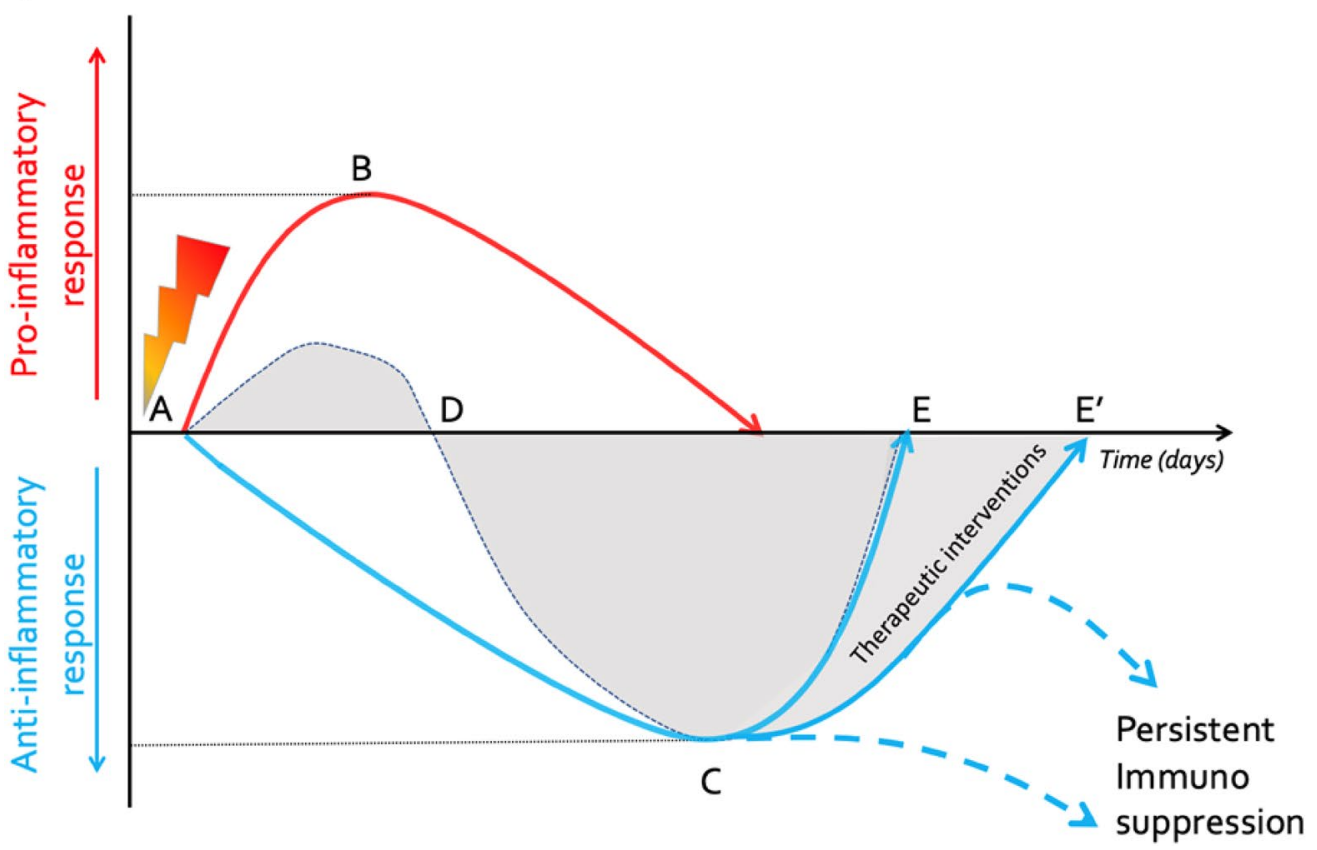

Fig. 2 Balance between the pro- and anti-inflammatory mediators. a Following an initial (infectious) insult (A), normally after correct (antibiotic) treatment the antagonistic forces of pro-inflammation (B), and anti-inflammation (C) regain balance (grey area shows net effects) maintaining healthy homeostasis (D), that will lead to recovery and survival (E). Adapted from Pfortmueller et al. with permission (Open Access CC BY Licence 4.0) Intensive Care Medicine Experimental (2017) 5:49 https://doi.org/10.1186/s40635-017-0163-0. b Following an (infectious) insult (A), during a dysregulated host response the pro-inflammatory (B) forces initially overwhelm anti-inflammation (C) resulting in an imbalance (D), followed by immunosuppression and increased anti-inflammatory mediators (grey area shows net effects). With different therapeutic interventions recovery (E) can be obtained or delayed ( $\left.E^{\prime}\right)$ or the patient can evolve into a state of persistent immunosupression or paralysis. Adapted from Pfortmueller et al. with permission (Open Access CC BY Licence 4.0) Intensive Care Medicine Experimental (2017) 5:49 https://doi.org/10.1186/s40635-017-0163-0 

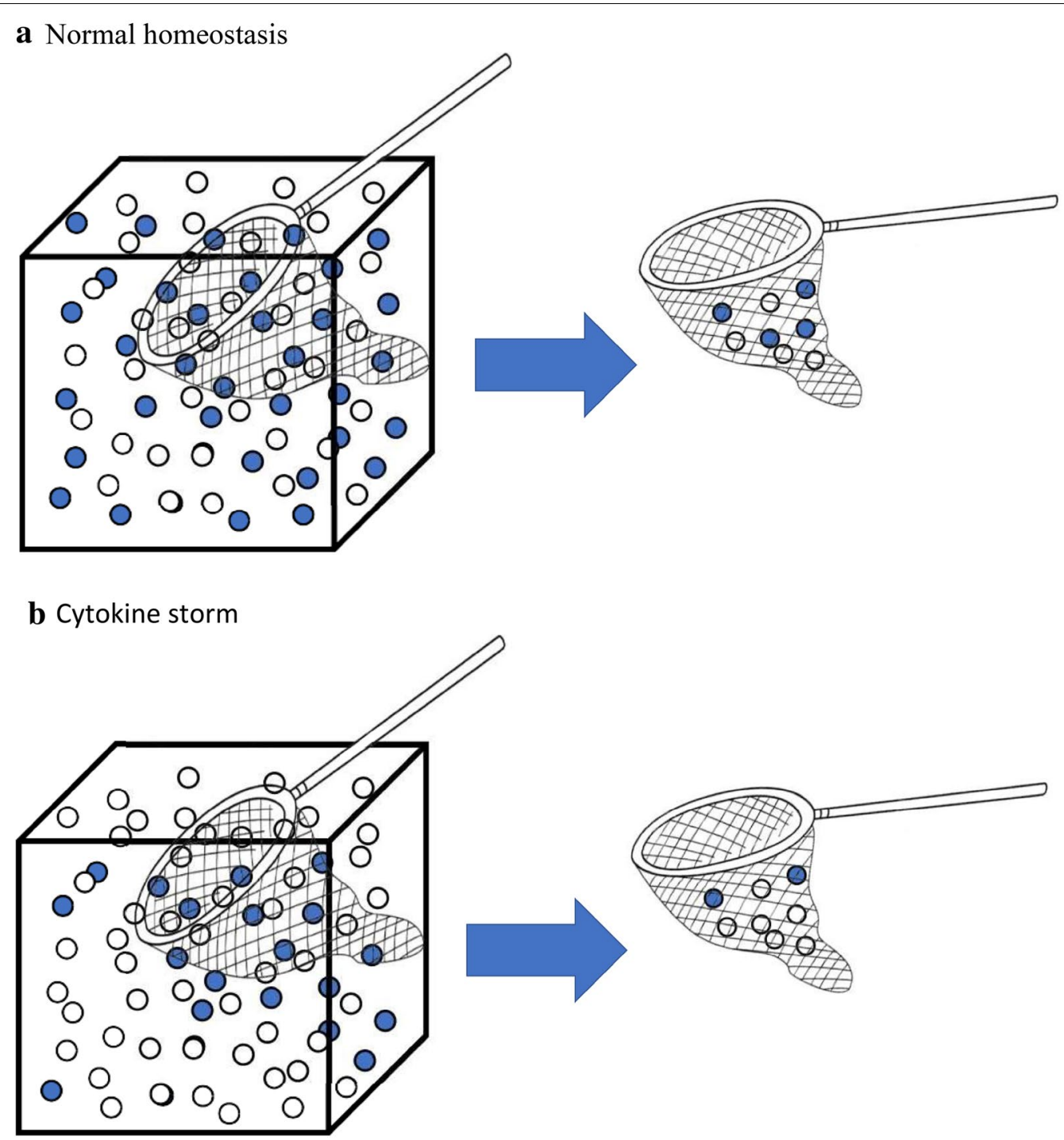

Fig. 3 The rationale of bulk removal of cytokines during cytokine storm. When homeostasis is normal, the pro-inflammatory (open circles), and anti-inflammatory (closed circles) mediators are in balance, molecules are present evenly as demonstrated in the first panel of the figure (a). When molecules are adsorbed in this scenario, the removal rate of both should be similar. However, if one component is in abundance-which is the case during cytokine storm $(\mathbf{b})$, then the removal rate should be proportionally higher from the mediator that is present in large numbers. This demonstrates the rationale why bulk removal of cytokines during cytokine storm may help to regain homeostatic balance

early observations have not translated into clinical benefit. Meta-analysis suggests that the only potentially effective BPT for the treatment of sepsis are plasma exchange or haemoadsorption particularly with Polymyxin B [2, 39]. Although initial results using Polymyxin $B$ haemoperfusion showed promise [31], this has not been borne out by subsequent randomized clinical trials [32]. Given the remit of sorbent technologies, we chose to focus on the use of CytoSorb as a haemoadsorption device. The adsorber has a surface of about $45,000 \mathrm{~m}^{2}$ compared to a conventional hemofilter with a surface of $1-1.5 \mathrm{~m}^{2}$ with a molecular cutoff of about $60 \mathrm{kDa}$ removing cytokines as well as other toxins and drugs. As a consequence, CytoSorb does not adsorb endotoxin which has a molecular weight of $100 \mathrm{kDa}$. CytoSorb is saturable regarding adsorption in the clinical setting (mostly after $8 \mathrm{~h}$ ) as evidenced by an rebound increase in the dose of vasopressors which can be tapered when changing the CytoSorb. So far, in vitro studies have not been evaluated regarding the adsorption-saturation process of adsorption. This could be an interesting area for future research. Multiple pre-clinical studies using animal models of sepsis have 
demonstrated reductions in various circulating cytokines and chemokines, reduced organ injury, and improved survival [61-64].

\section{Impact on different cytokines}

In a feasibility study, the removal of cytokines was confirmed with cytokine extraction rates approaching 30\% [65]. In this study, plasma concentrations of both IL-6 and TNF- $\alpha$, but not IL-10, were significantly reduced after the first hour of therapy [65]. Overall removal was greatest for IL-6, $28 \%(p=0.006)$, and least for tumour necrosis factor, $8.5 \%(p=0.13)$. However, plasma concentrations for all three cytokines increased over time and were above baseline by the end of the intervention $(4 \mathrm{~h})$. In a more recent study, the group of Antoine Schneider [66] conducted a single-centre pilot randomized controlled trial in 30 patients undergoing elective cardiac surgery and deemed at risk of complications. Patients were randomly allocated to either standard of care $(n=15)$ or CytoSorb ${ }^{\circledR}$ haemoadsorption $(n=15)$ during cardiopulmonary bypass $(\mathrm{CPB})$. The primary outcome was the difference between the two groups in cytokines levels (IL-1a, IL-1b, IL-2, IL-4, IL-5, IL-6, IL-10, TNF$\alpha$, interferon gamma (IFN- $\gamma$ ), monocyte chemotactic protein-1 (MCP-1) measured at anaesthesia induction, at the end of $\mathrm{CPB}$, sand 6 and $24 \mathrm{~h}$ post-CPB initiation. However, the intervention was associated neither with a decrease in pro- or anti-inflammatory cytokine levels nor with any improvement in relevant clinical outcomes [66]. These results are conflicting, and this is obviously a major concern regarding this new technique. So, altogether, no study using CytoSorb has shown a sustained plasma concentration reduction lasting during the whole treatment. Given the possibility that this technique may hold promise, we reviewed available data with the aim of producing current consensus statements and directing further research [67-69].

\section{Does the use of CytoSorb haemoadsorption therapy in patients with sepsis or septic shock demonstrate any clinical benefit?}

Although there is a significant body of experimental evidence supporting the potential use of haemoadsorption with CytoSorb in septic shock, there is far less evidence from human studies. There are over 100 case studies describing the use of CytoSorb in many clinical scenarios, and in general, the treatment is well tolerated, but clinical studies in general are small case series [70-72]. The largest study to date, a randomized controlled, open-label study enrolled patients from $10 \mathrm{Ger}-$ man study sites over the period 2008-2011 [73, 74]. A total of 582 patients were screened for this study of which 100 mechanically ventilated patients with severe sepsis or septic shock together with acute lung injury or acute respiratory distress syndrome were recruited. Final analysis was limited to 97 . Remarkably, no data regarding hemodynamic indices such as cardiac index or systemic vascular resistance was recorded. Patients were randomized initially on a 1:1 basis using sealed envelopes, but due to suspected irregularities, this system was changed after 32 patients were recruited to an electronic system using a block length of 6 . The treatment arm received $6 \mathrm{~h}$ of CytoSorb haemoperfusion per day for up to 7 days compared to standard care. The primary endpoint was a reduction in "normalized" rather than absolute IL- 6 levels with post-treatment IL- 6 levels divided by the individual patients' baseline IL-6 concentration. Unfortunately, in 22 patients, no valid primary endpoint was available leaving 75 patients in the final cohort. Secondary endpoints included a host of other cytokines, duration of mechanical ventilation, 28-day mortality and the MOD score. The primary endpoint of this study was negative. The use of haemoadsorption was not associated with a reduction in IL-6 levels. After adjusting for comorbidities (age, gender, and RRT), there was no association between treatment with haemoadsorption and mortality or indeed any of the other secondary endpoints. What was noted was that there were a larger number of clinically significant patients needing RRT in the treatment group (31.9\% vs $16.3 \%)$. Although the application of the CytoSorb cartridge did result in detectable IL-6 elimination (5-18\%) throughout the 6-h period, this did not translate to an overall reduction compared to control. This may appear incongruous as the effects of treatment on IL-6 between groups were measured at day 2 and not during the time periods employed to assess IL- 6 removal $(15,60,180$ and $360 \mathrm{~min}$, respectively) [73, 74].

Although this can be regarded as the first randomized controlled trial (RCT) employing the CytoSorb technique in sepsis, the results do not provide any evidence that treatment resulted in a clinically relevant endpoint. The study was powered for safety and efficacy of IL-6 removal. The patient population included was also less sick then in case series and in a recent registry [75], suggesting that since 2008, CytoSorb has been used in a sicker population. Similarly, a prospective randomized control trial in patients who underwent cardiac surgery also found no significant impact on cytokine concentrations although this study was not performed in sepsis [76]. In a recent propensity-matched retrospective study on 32 patients, the influence of intraoperative cytokine adsorption on the perioperative vasoplegia, inflammatory response and outcome during orthotopic heart transplantation was investigated [77]. Intraoperative CytoSorb treatment was associated with reduced vasopressor demand and less frequent RRT with no difference in length of mechanical 
ventilation and ICU stay [78]. Other case series in sepsis patients do point to a reduction in vasopressor dose, which may be considered a relevant endpoint, although these are not randomized studies [68-70]. The third interim analysis of the CytoSorb Registry [75] of 135 patients with septic shock and an acute physiology and chronic health evaluation II (APACHE-II) predicted mortality of $78 \%$ had an observed mortality of $65 \%$ with a marked reduction in both PCT and IL- 6 levels after $24 \mathrm{~h} \mathrm{[75].} \mathrm{Such} \mathrm{an} \mathrm{high} \mathrm{predicted} \mathrm{mortality} \mathrm{(78 \% )} \mathrm{can} \mathrm{be}$ explained by various reasons. First, it was recommended to only use Cytosorb haemoadsorption in patients with an APACHE-II score above 25. Second, clinicians were only starting the treatment as a salvage therapy, late in the course of the disease. Obviously, with a registry, you cannot control the eligibility criteria. Recently, the first randomized clinical trial on CytoSorb as a standalone (i.e.: without CRRT) haemoperfusion treatment in patients with septic shock, the ACESS trial (Adsorption of Cytokines Early in Septic Shock) was published [78, 79]. This was a proof of concept pilot study on 20 medical patients randomized into a CytoSorb and a standard treatment group, with cytokine adsorption initiated within the first $24 \mathrm{~h}$ after the onset of septic shock. The treatment proved to be safe and resulted in a significant reduction in norepinephrine requirement in the CytoSorb group as compared to controls: $\mathrm{T} 0=0.54[\mathrm{IQR}$ : $0.20-1.22], \quad \mathrm{T} 48=0.16[\mathrm{IQR}: \quad 0.07-0.48], \quad p=0.016$; Controls: $\mathrm{T} 0=0.43[\mathrm{IQR}: 0.19-0.64], \mathrm{T} 48=0.25[\mathrm{IQR}$ : $0.08-0.65] \mu \mathrm{g} / \mathrm{kg} / \mathrm{min}$. They also observed a significant reduction in procalcitonin (PCT): CytoSorb: T0 median $=20.6[\mathrm{IQR}: \quad 6.5-144.5], \quad \mathrm{T} 48=5.6[1.9-54.4]$, $p=0.004$; Control: $\mathrm{T} 0=13.2[7.6-47.8], \mathrm{T} 48=9.2[3.8-$ $44.2] \mathrm{ng} / \mathrm{mL}[78,79]$. The promising results of this pilot study may serve as the rationale of further large randomized clinical trials, but at present, there is a lack of robust evidence to support recommendations.

\section{Results of the consensus meeting}

To date, there is not enough data to provide any kind of evidence-based recommendations for the use of sorbent technologies, but the following points were considered by the panel as most important unanswered questions which may aid further recommendations.

\section{Point-1: Which patient would benefit the most from cytokine removal?}

The aim of any treatment is to select only patients who have a high probability of benefit from the intervention. To date, most of the case series included patients with a high predicted mortality in keeping with septic shock although few have included cytokine estimation [64, 71]. Where recorded, there is significant variation in
IL-6 levels, and therefore, one may conclude that some patients were treated where benefit would be negligible if indeed [IL-6] levels are predictive of response to therapy. Therefore, any future studies should attempt to enrich the study population through cytokine measurement or another marker of potential therapeutic response such as PCT [70]. A threshold PCT concentration may be included as an inclusion factor in future studies. Alternatively, patient selection may concentrate on those individuals who require high doses of vasopressor agents to maintain adequate organ perfusion as a reduction in such drugs is a meaningful clinical outcome which may translate into other benefits $[78,79]$.

Point-2: When to start cytokine removal therapy in sepsis? The timing of therapy remains an issue in many aspects of critical care medicine not least those involving extracorporeal therapies. Several of the case series report a less favourable outcome in those individuals who commenced treatment more than $24 \mathrm{~h}$ after diagnosis [70]. Data of the ACESS trial also support the concept of starting treatment within $24 \mathrm{~h}[78,79]$. It follows that any recommendation regarding timing of therapy will be dependent on the patient population and how prospective individuals are selected. Where the primary endpoint is a reduction in vasopressor, then timing will be relatively easy to define, whereas cytokine levels may be less clear especially given the differences in kinetics. Certainly, the rationale for any of the blood purification therapies would point to commencing treatment early in the disease process in order to maximize benefit. Maybe starting therapy directly in operating room during surgery for peritonitis patients with already hemodynamic impairment and catecholamine requirement should be considered for future studies. However, timing to intervene might be even more complex especially when sepsis-induced immunodepression is taken in account. This depression is essential to limit the consequences on the host tissues of the host response related to infection (i.e. maintain the inflammation-repair-healing cycle). Characterization of this immunodepression might be one of the best indicators to decide when to use adsorber technique to reduce inflammation, namely when the downregulation is modest or absent [22].

\section{Point-3: How long should cytokine removal therapy last and how long should it be continued?}

The optimum length of treatment is still undecided. Moreover, the length of time of initial therapy is also unclear. Schadler et al. treated patients for $6 \mathrm{~h}$ a day for 7 days [73, 74], whereas other reported studies either did not describe the treatment period or used the cartridge for $24 \mathrm{~h}[70,71]$. No evidence to date exists for the 
appropriate duration of adsorption therapy or indeed the initial treatment period. Again, this may be directed in future studies by the use of IL- 6 or rather PCT as a surrogate for total cytokine removal and reduction in systemic levels coupled with clinical improvement may be a logical place to start regarding treatment times. Also, it is unknown whether the initial therapy should be for 12 or $24 \mathrm{~h}$. Again, a pragmatic approach may be directed by cytokine estimation. Again, the study by Schadler [73, 74] may provide some answers. Although the 6-h treatment did not result in an overall reduction in IL- 6 compared to controls over a 24-h period, there was increased IL-6 elimination with the use of haemoadsorption [73, 74]. It follows that if the aim of treatment is to reduce cytokine levels, then a prolonged treatment period should be associated with a reduction in systemic levels. However, this removal in cytokines may be attenuated by cytokine shift from the interstitium into the blood compartment thereby negating any overall effect $[22,68,69]$. As a consequence, pharmacokinetic studies on cytokine clearance would be extremely useful to conduct in the future to better delineate the therapy duration of hemadsorption in relation to benefits for the patient with a cytokine storm.

\section{Point-4: Which patient population should be studied in the future?}

Future studies should aim to include as homogeneous cohorts of septic shock patients, especially those with very high vasopressor needs. Another interesting population to study may be patients with acute respiratory distress syndrome (ARDS). Mortality rate of ARDS still remains high between 30 and 50\% [80]. ARDS is a heterogeneous syndrome, characterized by increased pulmonary capillary permeability [81]. It is the accumulation of protein-rich fluid inside the alveoli that triggers damage to the capillary endothelium and alveolar epithelium leading to release of cytokines, producing diffuse alveolar damage [82]. Extracorporeal membrane oxygenation (ECMO) may serve as a salvage therapy with the CESAR trial that have led to an enhanced use of this therapy [83]. Several case reports mention the positive effect of incorporation of CysoSorb therapy for respiratory failure due to ARDS, using ECMO and CytoSorb therapy. The use of CytoSorb appeared to result in rapid resolution of neutropenia, reversal of toxic shock and rapid weaning of the high-dose vasopressor infusions and a significant reduction in the levels of circulating inflammatory mediators [84-88].
Point-5: What severity of sepsis would be the most appropriate to include in a study looking at cytokine removal therapy in patients with sepsis?

The intervention is more likely to benefit patients with high severity of illness (e.g. APACHE-II greater than 25) [75]. Refractory shock indicated by high doses of vasopressor support and early multiple organ failure with some evidence of a cytokine storm (i.e.: high PCT) should be preferred [70,71, 79].

Point-6: Which biomarker should be the most appropriate to include in a study looking at cytokine removal therapy in patients with septic shock?

It is uncertain whether cytokine concentrations can identify the ideal patient for treatment. Detecting persistent 'cytokine storm'-despite adequate resuscitation and source control-could be an alarming signal that cytokine removal may be beneficial. PCT as one of the most studied inflammatory biomarker in sepsis could be added as a biomarker to determine in which patient to start therapy $[87,88]$. It is difficult to determine the absolute cutoff values above which treatment may be indicated, but there is some evidence, that PCT kinetics, i.e. failure of PCT to decline or rapidly increasing levels may help to identify persisting cytokine storm [89]. It is indeed the reality that the molecular weight of PCT is about $13 \mathrm{kDa}$ and PCT might be removed by the adsorbent. Therefore, PCT is a good biomarker to decide when to start but not to follow the response to therapy nor to decide when to stop. IL-6 may also be a promising biomarker, but at present, little is known about IL-6 kinetics, and its response to appropriate or inappropriate treatment, which needs further research. Nevertheless, the biomarkers to characterize inflammatory status cannot be summarized by limited to IL-6 associated and PCT: we also do need to obtain information on cellular function (immune and tissular) [22].

\section{Point-7: Future research recommendations}

Based on the above, future studies must define the population in terms of physiological parameters (APACHE-II) or SOFA (Sequential Organ Failure Assessment) scores [90], vasopressor requirement, etc.), and there should be biomarker enrichment of patient selection probably using markers such as IL-6 or PCT levels. Secondly, clinically relevant endpoints should be selected. In the first instance, this may well be vasopressor dose under strict protocol guidance and adherence. In all cases, invasive hemodynamic monitoring including cardiac output and derived variables must be performed and data recorded, in order to have a better understanding on the pathophysiological effects of the therapy. With regard to 
treatment length, this should be guided by response in terms of other therapies as well as biomarker reduction if possible.

Regarding study endpoints, the consensus panel agreed that other endpoints rather than mortality should be chosen in the future to test the actual effect of this therapy. Monitoring organ dysfunction with measures like the SOFA score may be a better option, in order to understand the physiological effects of CytoSorb treatment. Furthermore, a hemodynamic primary endpoint such as the change in vasopressor need could also be applied, with mortality, ICU length of stay, length of mechanical ventilation, dialysis dependence, etc., as secondary endpoints. Finally, potential side effects should be also be evaluated like the removal of antibiotics, and especially lipophilic antibiotics ones [91].

\section{Conclusions}

To date, there is a paucity of data surrounding the use of haemoadsorption therapies in the treatment of septic shock. Although the consensus statements may appear somewhat uncertain, they reflect the current state of our understanding. Nevertheless, we hope that the current consensus paper also provides a framework for areas that need to be addressed in future work rather than continued reliance on single-centre case series.

For the time being, clinical results with the use of cytokine adsorbent therapies are scarce and rather disappointing. More studies should be performed to have a precise idea of adsorption properties (kinetics, saturability, potential mediator release, drug removal...) of the adsorbent. Plasma cytokine levels before and after treatment of various cytokines should be provided to clearly demonstrate the adsorptive properties. Only through well-conducted randomized controlled studies with appropriate patient selection criteria and endpoints of physiological relevance will we know whether haemoadsorption techniques are a future therapy for sepsis.

\footnotetext{
Abbreviations

APACHE-II: acute physiology and chronic health evaluation II; ARDS: acute respiratory distress syndrome; APCs: antigen-presenting cells; BPT: blood purification techniques; CPB: cardiopulmonary bypass; CRRT: continuous renal replacement therapies; $\mathrm{CVVH}$ : continuous veno-venous haemofiltration; CMT: cytokine modulatory techniques; $\mathrm{CHT}$ : $\mathrm{CytoSorb}^{\circledR}$, cytokine haemoadsorption techniques; DAMPs: damage-associated molecular patterns; EAA: endotoxin activity assay; ECMO: extracorporeal membrane oxygenation; GIPS: global increased permeability syndrome; HVHF: high-volume haemofiltration; ICU: intensive care unit; IFN- $\gamma$ : interferon gamma; IL: interleukin; IFN- $\gamma$ : interferon gamma; IFAD: international fluid academy day; MCP-1: monocyte chemotactic protein-1; MODS: multiple organ dysfunction score; PAMPs: pathogenassociated molecular patterns; PMX-HP: polymyxin B haemoperfusion; PCT: procalcitonin; PRCT: prospective randomized control trial; RCT: randomized control trial; RRT: renal replacement therapies; SAI: sepsis-associated immunosuppression; TNF-alpha: tumour necrosis factor alpha.
}

\section{Acknowledgements}

This article is endorsed by the International Fluid Academy (IFA). The mission statement of the IFA is to foster education, promote research on fluid management and hemodynamic monitoring, and thereby improve survival of critically ill by bringing together physicians, nurses, and others from throughout the world and from a variety of clinical disciplines. The IFA is integrated within the not-for-profit charitable organization iMERiT, International Medical Education and Research Initiative, under Belgian law. The IFA website (http://www.fluid academy.org) is now an official SMACC affiliated site (Social Media and Critical Care) and its content is based on the philosophy of FOAM (Free Open Access Medical education-\#FOAMed). The site recently received the HONcode quality label for medical education (https://www.healthonnet.org/HONcode/ Conduct.html?HONConduct519739). The consensus meeting was supported by an unrestricted educational grant by Cytosorbents that covered the costs for the travel and stay of the participants of the consensus meeting during the 6th IFAD. No extra fees nor honoraria were paid. The company was not involved during the writing process of this executive summary.

\section{Authors' contributions}

$\mathrm{PMH}, \mathrm{LF}, \mathrm{EH}$ and $\mathrm{ZM}$ designed the paper and wrote the first draft. All authors participated in drafting and reviewing. All authors read and approved the final manuscript.

\section{Funding}

None.

\section{Availability of data and materials}

Not applicable.

\section{Ethics approval and consent to participate}

Not applicable.

\section{Consent for publication \\ Not applicable.}

\section{Competing interests}

Patrick M. Honore has served as consultant for Baxter, Gambro, Nikkiso and Asahi. He received honoraria for lectures from Baxter, Gambro, Nikkiso, Asahi, BBraun and Cytosorbents. Manu L.N.G. Malbrain is inaugural President of WSACS (The Abdominal Compartment Society, http://www.wsacs.org) and current Treasurer. He is also co-founder of WSACS. He is also member of the medical advisory Board of Getinge (Pulsion Medical Systems) and Serenno Medical. He consults for Baxter, Maltron, ConvaTec, Acelity, Spiegelberg and Holtech Medical. He is co-founder of the International Fluid Academy (IFA, http://www.fluidacademy.org). Zolt Molnar recived honoratia for lectures from Cytosorbents. Olivier Joannes-Boyau has served as a consultant to BBraun and Baxter. Received honoraria or travel expenses for lectures from Baxter, Fresenius and BBraun. Rita Jacobs has no conflicts of interest with this paper. Eric Hoste has no conflicts of interest with this paper. Lui Forni has no conflict of interests with this paper.

\section{Author details}

${ }^{1}$ Intensive Care Department, CHU Brugmann University Hospital, 4, Place Arthur Van Gehuchtenplein, 1020 Brussels, Belgium. ${ }^{2}$ Intensive Care Department, Ghent University Hospital, Ghent, Belgium. ${ }^{3}$ Department of Anaesthesiology and Intensive Therapy, Faculty of Medicine, University of Szeged, Szeged, Hungary. ${ }^{4}$ Intensive Care Department, University Hospital Brussels (UZB), Jette, Belgium. ${ }^{5}$ Département d'Anesthésie-Réanimation SUD, CHU Bordeaux, 33000 Bordeaux, France. ${ }^{6}$ Faculty of Medicine and Pharmacy, Vrije Universiteit Brussel (VUB), Brussels, Belgium. ${ }^{7}$ Department of Critical Care, Royal Surrey County Hospital, NHS Foundation Trust, Guildford, UK. ${ }^{8}$ Department of Clinical and Experimental Medicine, Faculty of Health Sciences, University of Surrey, Guildford, UK.

Received: 30 January 2019 Accepted: 7 May 2019

Published online: 14 May 2019 


\section{References}

1. Rhee C, Dantes R, Epstein L, CDC prevention epicenter program, et al. Incidence and trends of sepsis in US Hospitals using clinical vs claims data, 2009-2014. JAMA. 2017;318:1241-9.

2. Rimmer $\mathrm{E}$, Houston BL, Kumar A, et al. The efficacy and safety of plasma exchange in patients with sepsis and septic shock: a systematic review and meta-analysis. Crit Care. 2014;18:699.

3. Stoller J, Halpin L, Weis M, et al. Epidemiology of severe sepsis: 20082012. J Crit Care. 2016:31(1):58-62.

4. Machado FR, Cavalcanti AB, Bozza FA, SPREAD Investigators; Latin American Sepsis Institute Network, et al. The epidemiology of sepsis in Brazilian intensive care units (the Sepsis PREvalence Assessment Database, SPREAD): an observational study. Lancet Infect Dis. 2017:17:1180-9.

5. Shankar-Hari M, Ambler M, Mahalingasivam V, Jones A, Rowan K, Rubenfeld GD. Evidence for a causal link between sepsis and long-term mortality: a systematic review of epidemiologic studies. Crit Care. 2016;20:101.

6. Harrison AM, Gajic O, Pickering BW, Herasevich V. Development and implementation of sepsis alert systems. Clin Chest Med. 2016;37:219-29.

7. Gaieski DF, Edwards JM, Kallan MJ, Carr BG. Benchmarking the incidence and mortality of severe sepsis in the United States. Crit Care Med. 2013;41:1167-74

8. Kaukonen KM, Bailey M, Suzuki S, Pilcher D, Bellomo R. Mortality related to severe sepsis and septic shock among critically ill patients in Australia and New Zealand, 2000-2012. JAMA. 2014;311:1308-16.

9. Angus DC, van der Poll T. Severe sepsis and septic shock. N Engl J Med. 2013;369:840-51

10. Hotchkiss RS, Monneret G, Payen D. Sepsis-induced immunosuppression: from cellular dysfunctions to immunotherapy. Nat Rev Immunol. 2013;13:862-74.

11. Iskander KN, Osuchowski MF, Stearns-Kurosawa DJ, et al. Sepsis: multiple abnormalities, heterogeneous responses, and evolving understanding. Physiol Rev. 2013;93:1247-88.

12. Dreiher J, Almog Y, Sprung CL, SEPSIS-ISR Group, et al. Temporal trends in patient characteristics and survival of intensive care admissions with sepsis: a multicenter analysis. Crit Care Med. 2012;40(3):855-60.

13. Kadri SS, Rhee C, Strich JR, et al. Estimating ten-year trends in septic shock incidence and mortality in United States academic medical centers using clinical data. Chest. 2017:151:278-85.

14. Stevenson EK, Rubenstein AR, Radin GT, Wiener RS, Walkey A. Two decades of mortality trends among patients with severe sepsis: a comparative meta-analysis. Crit Care Med. 2014;42:625-31.

15. Iwashyna TJ, Cooke CR, Wunsch H, Kahn JM. Population burden of longterm survivorship after severe sepsis in older Americans. J Am Geriatr Soc. 2012;60:1070-7.

16. Torio CM, Andrews RM. National inpatient hospital costs: the most expensive conditions by payer, 2011: statistical brief \#160 Healthcare Cost and Utilization Project (HCUP) Statistical Briefs. Rockville (MD); 2006.

17. Dellinger RP, Levy MM, Rhodes A, et al. Surviving sepsis campaign: international guidelines for management of severe sepsis and septic shock, 2012. Intensive Care Med. 2013;39:165-228.

18. Zhou F, Peng Z, Murugan R, Kellum JA. Blood purification and mortality in sepsis: a meta-analysis of randomized trials. Crit Care Med. 2013;41:2209-20.

19. Callard R, George AJ, Stark J. Cytokines, chaos, and complexity. Immunity. 1999:11:507-13.

20. Singer M, Deutschman CS, Seymour CW, et al. The third international consensus definitions for sepsis and septic shock (sepsis-3). JAMA 2016:315(8):801-10. https://doi.org/10.1001/jama.2016.0287.

21. Ronco C, Tetta C, Mariano F, et al. Interpreting the mechanisms of continuous renal replacement therapy in sepsis: the peak concentration hypothesis. Artif Organs. 2003;27(9):792-801

22. van der Poll T, van de Veerdonk FL, Scicluna BP, Netea MG. The immunopathology of sepsis and potential therapeutic targets. Nat Rev Immunol. 2017:17(7):407-20. https://doi.org/10.1038/nri.2017.36.

23. Honoré PM, Matson JR. Extracorporeal removal for sepsis: acting at the tissue level-the beginning of a new era for this treatment modality in septic shock. Crit Care Med. 2004;32(3):896-7.

24. Di Carlo JV, Alexander SR. Hemofiltration for cytokine-driven illnesses: the mediator delivery hypothesis. Int J Artif Organs. 2005;28:777-86.
25. Peng ZY, Bishop JV, Wen XY, et al. Modulation of chemokine gradients by apheresis redirects leukocyte trafficking to different compartments during sepsis, studies in a rat model. Crit Care. 2014;18:R141.

26. Lukaszewicz AC, Payen D. Purification methods: a way to treat severe acute inflammation related to sepsis? Crit Care. 2013;17:159.

27. Cole L, Bellomo R, Journois D, Davenport P, Baldwin I, Tipping P. Highvolume haemofiltration in human septic shock. Intensive Care Med. 2001;27:978-86.

28. Honore PM, Jamez J, Wauthier $M$, et al. Prospective evaluation of shortterm, high-volume isovolemic hemofiltration on the hemodynamic course and outcome in patients with intractable circulatory failure resulting from septic shock. Crit Care Med. 2000;28:3581-7.

29. Joannes-Boyau O, Honore PM, Perez P, et al. High-volume versus standard-volume haemofiltration for septic shock patients with acute kidney injury (IVOIRE study): a multicentre randomized controlled trial. Intensive Care Med. 2013;39:1535-46.

30. Clark E, Molnar AO, Joannes-Boyau O, Honore PM, Sikora L, Bagshaw SM High-volume hemofiltration for septic acute kidney injury: a systematic review and meta-analysis. Crit Care. 2014;18:R7.

31. Cavaillon JM, Munoz C, Fitting C, Misset B, Carlet J. Circulating cytokines: the tip of the iceberg? Circ Shock. 1992;38(2):145-52.

32. Sun S, Sursal T, Adibnia Y, Zhao C, Zheng Y, Li H, LE Otterbein, et al. Mitochondrial DAMPs increase endothelial permeability through neutrophil dependent and independent pathways. PLoS ONE. 2013;8(3):e59989. https://doi.org/10.1371/journal.pone.0059989.

33. Vincent JL, Laterre PF, Cohen J, et al. A pilot-controlled study of a polymyxin B-immobilized hemoperfusion cartridge in patients with severe sepsis secondary to intra-abdominal infection. Shock. 2005;23:400-5.

34. Cruz DN, Antonelli M, Fumagalli R, et al. Early use of polymyxin B hemoperfusion in abdominal septic shock: the EUPHAS randomized controlled trial. JAMA. 2009:301:2445-52.

35. Payen DM, Guilhot J, Launey Y, ABDOMIX Group, et al. Early use of polymyxin $B$ hemoperfusion in patients with septic shock due to peritonitis: a multicenter randomized control trial. Intensive Care Med. 2015:41:975-84.

36. Coudroy R, Payen D, Launey Y, ABDOMIX group, et al. Modulation by polymyxin-B hemoperfusion of inflammatory response related to severe peritonitis. Shock. 2017:47:93.

37. Dellinger RP, Bagshaw SM, Antonelli M, Foster DM, Klein DJ, Marshall JC, et al. Effect of targeted polymyxin B hemoperfusion on 28-day mortality in patients with septic shock and elevated endotoxin level: the EUPHRATES randomized clinical trial. JAMA. 2018;320(14):1455-63. https://doi. org/10.1001/jama.2018.14618.

38. Iba T, Fowler L. Is polymyxin B-immobilized fiber column ineffective for septic shock? A discussion on the press release for EUPHRATES trial. J Intensive Care. 2017;5:40.

39. Chang T, Tu Y-K, Lee C-T, et al. Effects of polymyxin B hemoperfusion on mortality in patients with severe sepsis and septic shock: a systemic review, meta-analysis update, and disease severity subgroup meta-analysis. Crit Care Med. 2017;45:e858-64.

40. Payen D. Haemoperfusion with polymyxin B membrane: recent results for an old debate! Anaesth Crit Care Pain Med. 2019;38(1):3-4. https://doi. org/10.1016/j.accpm.2018.12.010.

41. Pickkers P, Russell JA. Treatment with a polymyxin B filter to capture endotoxin in sepsis patients: is there a signal for therapeutic efficacy? Intensive Care Med. 2019;45(2):282-3. https://doi.org/10.1007/s00134-018-5481-5.

42. Duchesne JC, Kaplan LJ, Balogh ZJ, Malbrain MNLG. Role of permissive hypotension, hypertonic resuscitation and the global increased permeability syndrome in patients with severe haemorrhage: adjuncts to damage control resuscitation to prevent intra-abdominal hypertension. Anaesthesiol Intensive Ther. 2015;47:143-55

43. Malbrain MLNG, Van Regenmortel N, Saugel B, et al. Principles of fluid management and stewardship in septic shock: it is time to consider the four D's and the four phases of fluid therapy. Ann Intensive Care. 2018;8(1):66. https://doi.org/10.1186/s13613-018-0402-.

44. Gruda MC, Ruggeberg KG, O'Sullivan P, et al. Broad adsorption of sepsisrelated PAMP and DAMP molecules, mycotoxins, and cytokines from whole blood using CytoSorb ${ }^{\circledR}$ sorbent porous polymer beads. PLOS ONE. 2018;13:e0191676.

45. Malbrain ML, Marik PE, Witters I, et al. Fluid overload, de-resuscitation, and outcomes in critically ill or injured patients: a systematic review 
with suggestions for clinical practice. Anaesthesiol Intensive Ther. 2014;46(5):361-80. https://doi.org/10.5603/ait.2014.0060.

46. Kobe Y, Oda S, Matsuda K, Nakamura M, Hirasawa H. Direct hemoperfusion with a cytokine-adsorbing device for the treatment of persistent or severe hypercytokinemia: a pilot study. Blood Purif. 2007;25:446-53.

47. Pfortmueller CA, Meisel C, Fux M, Schefold JC. Assessment of immune organ dysfunction in critical illness: utility of innate immune response markers. Intensive Care Med Exp. 2017;5:49.

48. Hotchkiss RS, Monneret G, Payen D. Immunosuppression in sepsis: a novel understanding of the disorder and a new therapeutic approach. Lancet Infect Dis. 2013;13:260-8.

49. Monneret G, Venet F, Pachot A, Lepape A. Monitoring immune dysfunctions in the septic patient: a new skin for the old ceremony. Mol Med. 2008;14:64-78.

50. Boomer JS, To K, Chang KC, et al. Immunosuppression in patients who die of sepsis and multiple organ failure. JAMA. 2011;306:2594-605.

51. Schefold JC. Measurement of monocytic HLA-DR (mHLA-DR) expression in patients with severe sepsis and septic shock: assessment of immune organ failure. Intensive Care Med. 2010;36:1810-2.

52. Leentjens J, Kox M, Koch RM, et al. Reversal of immunoparalysis in humans in vivo: a double-blind, placebo-controlled, randomized pilot study. Am J Respir Crit Care Med. 2012;186:838-45.

53. Drewry AM, Ablordeppey EA, Murray ET, et al. Comparison of monocyte human leukocyte antigen-DR expression and stimulated tumor necrosis factor alpha production as outcome predictors in severe sepsis: a prospective observational study. Crit Care. 2016;20:334.

54. Quinto BM, lizuka IJ, Monte JC, et al. TNF-a depuration is a predictor of mortality in critically ill patients under continuous veno-venous hemodiafiltration treatment. Cytokine. 2015;71(2):255-60. https://doi. org/10.1016/j.cyto.2014.10.024.

55. Van Snick J. Interleukin-6: an overview. Annu Rev Immunol. 1990;8:253-78.

56. Venet F, Lukaszewicz AC, Payen D, Hotchkiss R, Monneret G. Monitoring the immune response in sepsis: a rational approach to administration of immunoadjuvant therapies. Curr Opin Immunol. 2013;25:477-83.

57. Monneret $G$, Finck $M E$, Venet $F$, et al. The anti-inflammatory response dominates after septic shock: association of low monocyte HLA-DR expression and high interleukin-10 concentration. Immunol Lett. 2004;95:193-8.

58. Frencken JF, van Vught LA, Peelen LM, et al. An unbalanced inflammatory cytokine response is not associated with mortality following sepsis: a prospective cohort study. Crit Care Med. 2017:45:e493-9.

59. Kellum JA, Kong L, Fink MP, et al. Understanding the inflammatory cytokine response in pneumonia and sepsis: results of the genetic and inflammatory markers of sepsis (GenIMS) study. Arch Intern Med. 2007:167:1655-63.

60. De Vriese AS, Colardyn FA, Philippe JJ, Vanholder RC, De Sutter JH, Lameire NH. Cytokine removal during continuous hemofiltration in septic patients. J Am Soc Nephrol. 1999;10:846-53.

61. Kellum JA, Song M, Venkataraman R. Hemoadsorption removes tumor necrosis factor, interleukin-6, and interleukin-10, reduces nuclear factorkappaB DNA binding, and improves short-term survival in lethal endotoxemia. Crit Care Med. 2004;32:801-5.

62. Namas RA, Namas R, Lagoa C, et al. Hemoadsorption reprograms inflammation in experimental gram-negative septic peritonitis: insights from in vivo and in silico studies. Mol Med. 2012;18:1366-74.

63. Peng ZY, Carter MJ, Kellum JA. Effects of hemoadsorption on cytokine removal and short-term survival in septic rats. Crit Care Med. 2008;36:1573-7.

64. Peng ZY, Wang HZ, Carter MJ, et al. Acute removal of common sepsis mediators does not explain the effects of extracorporeal blood purification in experimental sepsis. Kidney Int. 2012;81:363-9.

65. Kellum JA, Venkataraman R, Powner D, Elder M, Hergenroeder G, Carter M. Feasibility study of cytokine removal by hemoadsorption in brain-dead humans. Crit Care Med. 2008;36:268-72.

66. Poli EC, Alberio L, Bauer-Doerries A, Marcucci C, Roumy A, Kirsch M, et al. Cytokine clearance with CytoSorb ${ }^{\circledR}$ during cardiac surgery: a pilot randomized controlled trial. Crit Care. 2019;23(1):108. https://doi.org/10.1186/ s13054-019-2399-4.

67. Sathe PSP, Kumar S, Choudhary S. Clinical experience of using a novel extracorporeal cytokine adsorption column for treatment of septic shock with multiorgan failure. Crit Care (London, England). 2015;19(Suppl 1):P130.

68. Honoré PM, Matson JR. Hemofiltration, adsorption, sieving and the challenge of sepsis therapy design. Crit Care. 2002;6:394-6 (Epub 2002 Sep 4. Review).

69. Matson J, Zydney A, Honoré PM. Blood filtration: new opportunities and the implications of systems biology. Crit Care Resusc. 2004;6:209-17.

70. DA Laddomada T, Balicco B. Case series of patients with severe sepsis and septic shock treated with a new extracorporeal sorbent. Crit care (London, England). 2016;20:P193.

71. Kogelmann K, Jarczak D, Scheller M, Druner M. Hemoadsorption by CytoSorb in septic patients: a case series. Crit Care. 2017;21:74.

72. Friesecke S, Stecher SS, Gross S, Felix SB, Nierhaus A. Extracorporeal cytokine elimination as rescue therapy in refractory septic shock: a prospective single-center study. J Artif Organs. 2017;20:252-9.

73. Schädler D, Porzelius $C$, Jörres $A$, et al. A multicenter randomized controlled study of an extracorporeal cytokine hemoadsorption device in septic patients. Crit Care. 2013;17(Suppl 2):P62

74. Schadler D, Pausch C, Heise D, et al. The effect of a novel extracorporeal cytokine hemoadsorption device on IL-6 elimination in septic patients: a randomized controlled trial. PLoS ONE. 2017;12:e0187015.

75. Friesecke S, Träger K, Schittek GA, et al. International registry on the use of the CytoSorb ${ }^{\circledR}$ adsorber in ICU patients: study protocol and preliminary results. Med Klin Intensivmed Notfmed. 2017. https://doi.org/10.1007/ s00063-017-0342-5.

76. Bernardi MH, Rinoesl H, Dragosits K, et al. Effect of hemoadsorption during cardiopulmonary bypass surgery_-a blinded, randomized, controlled pilot study using a novel adsorbent. Crit Care. 2016;20:96.

77. Nemeth E, Kovacs E, Racz K, et al. Impact of intraoperative cytokine adsorption on outcome of patients undergoing orthotopic heart transplantation—an observational study. Clin Transpl. 2018. https://doi. org/10.1111/ctr.13211.

78. Öveges N, Hawchar F, László l, et al. Early cytokine adsorption in septic shock (ACESS-trial): results of a proof concept, pilot study. Crit Care. 2018:22(Suppl 1):82.

79. Hawchar F, Lazlo I, Oveges N, Trasy D, Molnar Z. Extracorporeal cytokine adsorption in septic shock: a proof of concept randomized, controlled pilot study. J Crit Care. 2018. https://doi.org/10.1016/j.jcrc.2018.11.003.

80. Bellani G, Laffey JG, Pham T, LUNG SAFE Investigators; ESICM Trials Group, et al. Epidemiology, patterns of care, and mortality for patients with acute respiratory distress syndrome in intensive care units in 50 countries. JAMA. 2016:315(8):788-800

81. Umbrello M, Formenti P, Bolgiaghi LU, Chiumello D. Current concepts of ARDS: a narrative review. Int J Mol Sci. 2017;18:64.

82. Martin TR. Lung cytokines and ARDS: Roger S Mitchell lecture. Chest. 1999;116(1 Suppl):2S-8S.

83. Peek GJ, Mugford M, Tiruvoipati R. Efficacy and economic assessment of conventional ventilatory support versus extracorporeal membrane oxygenation for severe adult respiratory failure (CESAR): a multicentre randomised controlled trial. Lancet. 2009;374:1351-63.

84. Lees NJ, Rosenberg A, Hurtado-Doce Al, et al. Combination of ECMO and cytokine adsorption therapy for severe sepsis with cardiogenic shock and ARDS due to Panton-Valentine leucocidin-positive Staphylococcus aureus pneumonia and H1N1. J Artif Organs. 2016;19(4):399-402.

85. Traeger K, Schuetz C, Fischer G, et al. Cytokine reduction in the setting of an ARDS-associated inflammatory response with multiple organ failure. In: Case reports in critical care.

86. Bruenger F, Kizner L, Weile J, Morshuis M, Gummert JF. First successful combination of ECMO with cytokine removal therapy in cardiogenic septic shock: a case report. Int J Artif Organs. 2015;30:113-6.

87. Becze Z, Molnar Z, Fazakas J. Can procalcitonin levels indicate the need for adjunctive therapy in sepsis? Int J Antimicrob Agents. 2015;46(Suppl 1):S13-8.

88. Pupelis G, Drozdova N, Mukans M, Malbrain ML. Serum procalcitonin is a sensitive marker for septic shock and mortality in secondary peritonitis. Inflammation. 2014;37(1):223-34. https://doi.org/10.1007/s1075 3-013-9733-9.

89. Trásy D, Tánczos K, Németh M, EProK study group, et al. Early procalcitonin kinetics and appropriateness of empirical antimicrobial therapy in critically ill patients: a prospective observational study. J Crit Care. 2016;34:50-5. https://doi.org/10.1016/j.jcrc.2016.04.007. 
90. Vincent JL, de Mendonça A, Cantraine F, et al. Use of the SOFA score to assess the incidence of organ dysfunction/failure in intensive care units: results of a multicenter, prospective study. Working group on "sepsisrelated problems" of the European Society of Intensive Care Medicine. Crit Care Med. 1998;26:1793-800.

91. Poli EC, Simoni C, André P, Buclin T, Longchamp D, Perez MHet al. Clindamycin clearance during Cytosorb ${ }^{\circledR}$ hemoadsorption: a case report and pharmacokinetic study. Int J Artif Organs. 2019;391398819831303. https ://doi.org/10.1177/0391398819831303 [Epub ahead of print].

\section{Publisher's Note}

Springer Nature remains neutral with regard to jurisdictional claims in published maps and institutional affiliations.

\section{Submit your manuscript to a SpringerOpen ${ }^{\odot}$ journal and benefit from:}

- Convenient online submission

- Rigorous peer review

- Open access: articles freely available online

- High visibility within the field

- Retaining the copyright to your article

Submit your next manuscript at $\boldsymbol{\nabla}$ springeropen.com 